\title{
Cosmic Chronometers: Constraining the Equation of State of Dark Energy. I: $H(z)$ Measurements
}

\author{
Daniel Stern ${ }^{1}$, Raul Jimenez ${ }^{2}$, Licia Verde ${ }^{2}$, Marc \\ Kamionkowski ${ }^{3}$ \& S. Adam Stanford ${ }^{4}$ \\ ${ }^{1}$ Jet Propulsion Laboratory, California Institute of Technology, Mail \\ Stop 169-506, Pasadena CA-91109, USA \\ ${ }^{2}$ ICREA 83 Institute of Sciences of the Cosmos (ICC), University of \\ Barcelona, Barcelona 08028, Spain \\ ${ }^{3}$ California Institute of Technology, Mail Code 350-17, Pasadena, \\ California 91125, USA \\ ${ }^{4}$ University of California, Davis, CA 95616 and Institute for \\ Geophysics and Planetary Physics, Lawrence Livermore National \\ Laboratory, Livermore, CA 94551
}

E-mail: stern@thisvi.jpl.nasa.gov, raul@icc.ub.edu, licia@icc.ub.edu, kamion@tapir.caltech.edu, stanford@physics.ucdavis.edu

\begin{abstract}
We present new determinations of the cosmic expansion history from red-envelope galaxies. We have obtained for this purpose high-quality spectra with the Keck-LRIS spectrograph of red-envelope galaxies in 24 galaxy clusters in the redshift range $0.2<z<1.0$. We complement these Keck spectra with high-quality, publicly available archival spectra from the SPICES and VVDS surveys. We improve over our previous expansion history measurements in Simon et al. (2005) by providing two new determinations of the expansion history: $H(z)=97 \pm 62 \mathrm{~km} \mathrm{sec}^{-1} \mathrm{Mpc}^{-1}$ at $z \simeq 0.5$ and $H(z)=90 \pm 40 \mathrm{~km} \mathrm{sec}^{-1} \mathrm{Mpc}^{-1}$ at $z \simeq 0.8$. We discuss the uncertainty in the expansion history determination that arises from uncertainties in the synthetic stellar-population models. We then use these new measurements in concert with cosmic-microwave-background (CMB) measurements to constrain cosmological parameters, with a special emphasis on dark-energy parameters and constraints to the curvature. In particular, we demonstrate the usefulness of direct $H(z)$ measurements by constraining the darkenergy equation of state parameterized by $w_{0}$ and $w_{a}$ and allowing for arbitrary curvature. Further, we also constrain, using only CMB and $H(z)$ data, the number of relativistic degrees of freedom to be $4 \pm 0.5$ and their total mass to be $<0.2 \mathrm{eV}$, both at $1 \sigma$.
\end{abstract}

\section{Introduction}

Direct supernova measurements of the deceleration parameter [1], as well as indirect measurements based upon a combination of results from the cosmic microwave background (CMB) 2], large-scale structure (LSS) 3, 4, and the Hubble constant [5] indicate that the expansion is accelerating. This suggests either that gravity on the largest scales is described by some theory other than general relativity and/or that the Universe is filled with some sort of negative-pressure "dark energy" that 
drives the accelerated expansion [6]; either way, it requires new physics beyond general relativity and the standard model of particle physics. These observations have garnered considerable theoretical attention as well as observational and experimental efforts to learn more about the new physics coming into play.

The simplest possibility is to extend Einstein's equation with a cosmological constant, or equivalently, to hypothesize a fluid with an equation-of-state parameter $w \equiv p / \rho=-1$ (with $p$ and $\rho$ the pressure and energy density, respectively). However, it may well be that the cosmological "constant" actually evolves with time, in which case $w \neq-1$, and there are a variety of theoretical reasons [7] to believe that this is the case. Precise measurement of $w(z)$ (with, in general, a parameterized redshift dependence) or, equivalently, the cosmic expansion history, has thus become a central goal of physical cosmology [8, 10].

Among the techniques to determine the cosmic expansion history are supernova searches, baryon acoustic oscillations (BAO) [11, 12, 14, 15, weak lensing [17, and galaxy clusters [16]. These techniques all have different strengths, and they all also suffer from a different set of weaknesses. As argued in the ESO/ESA and Dark Energy Task Force reports [8, 10, robust conclusions about the cosmic expansion history will likely require several avenues to allow for cross checks. There may also still be room for other ideas for determining the expansion history.

A common weakness of supernova searches, BAO (at least the angular clustering), weak lensing, and cluster-based measurements is that they rely largely on integrated quantities. For example supernovae probe the luminosity distance,

$$
d_{L}(z)=(1+z) \int_{z}^{0}\left(1+z^{\prime}\right) \frac{d t}{d z^{\prime}} d z^{\prime}
$$

The other probes rely on similar quantities, which depend on an integral of the expansion history, to determine the expansion history, rather than the expansion history itself. The purpose of the differential-age technique [18 is to circumvent this limitation by measuring directly the integrand, $d t / d z$, or in other words, the change in the age of the Universe as a function of redshift. This can be achieved by measuring ages of galaxies with respect to a fiducial model, thus circumventing the need to compute absolute ages. From Galactic globular clusters age-dating we know that relative ages are much more accurately determined than absolute ages (e.g., Refs. [19, 20, 21]). A preliminary analysis, with archival data, has already been carried out [22, 23], and the results applied to constrain dark-energy theories (e.g., Refs. [24]).

The challenge with the differential-age measurement is to find a population of standard clocks and accurately date them. There is now growing observational evidence that the most massive galaxies contain the oldest stellar populations up to redshifts of $z \sim 1-2$ [25, 26, 27, 28, 29]. Refs. 28] and [30] have shown that the most massive galaxies have less than $1 \%$ of their present stellar mass formed at $z<1$. Ref. [29] shows that star formation in massive systems in high-density regions - i.e., galaxy clusters - ceased by redshift $z \sim 3$ and Ref. [31] shows that massive systems, those with stellar masses $>5 \times 10^{11} \mathrm{M}_{\odot}$, have finished their star-formation activity by $z \sim 2$. There is thus considerable empirical evidence for a population of galaxies, harbored in the highest-density regions of galaxy clusters, that has formed its stellar population at high redshift, $z>2$, and that since that thime this population has been evolving passively, without further episodes of star formation. These galaxies trace the "red envelope," and are the oldest objects in the Universe at every redshift. The 
differential ages of these galaxies should thus be a good indicator for the rate of change of the age of the Universe as a function of redshift.

Here we report the first results of an observational campaign to obtain high signalto-noise Keck spectra of red-envelope galaxies in a number of galaxy clusters at redshift $z<1$.0. In particular, we report spectroscopy for galaxies in 24 rich galaxy clusters. These new data extend earlier results from archival field galaxy data at $z<0.5$ [22, 23] to the redshift range $0.5<z<1$ crucial for determining $w$. They also allow us to probe massive galaxies in clusters, which are older, and thus provide a much more efficient avenue to determine the age at any given redshift.

Our paper is organized as follows. We first estimate in 22 the statistical uncertainties in the age determination that arise from noisy spectra and finite sample sizes. The analysis demonstrates that the age and metallicity of galaxies can be disentangled from the measured spectra given high enough signal-to-noise, wide enough spectral coverage and accurate modeling, and it helps identify the wavelength ranges required to optimize the age determination. These arguments motivate the Keck observations we have made. We discuss the stellar-population models in $₫ 3$ and estimate the uncertainties in the age determination that arise from uncertainties in the stellar-population models. Section 4 discusses the observations and the galaxy spectra used in our analysis. These include both new spectra obtained at Keck (described in more detail and catalogued in Paper II, 9]), as well as galaxy spectra from archival data. Section 5 presents results for our measurement of the age-redshift relation, or equivalently, for the expansion rate $H(z)$ as a function of $z$. In 96 , we combine our measurements of $H(z)$ with $\mathrm{CMB}$ data to derive constraints to cosmological parameters. We conclude in $\S 7$.

\section{Statistical Uncertainties in the Age Reconstruction}

We first quantify the statistical error, arising from noisy spectra and from finite sample sizes, in the age determination. To do so we assume that our theoretical stellar models are an accurate representation of reality and then recover the galaxy age and metallicity from a spectrum over a given wavelength range and with a given level of photon noise. We have thus generated a series of mock spectra using the Charlot \& Bruzual (2007) (CB08, Charlot private communication) stellar-population models with random noise for different values of signal-to-noise and for different spectral coverages. In Fig. 1 we explore how well we recover the age of the input spectrum for a signal-tonoise $S / N=10$ per resolution element of $3 \AA$. The age range is divided into 60 equally spaced bins in age from 1 to $14 \mathrm{Gyr}$, and 25 equally spaced bins in metallicity from from 0.2 to $2.5 Z_{\odot}$. In each bin we generate 100 Monte Carlo realizations of the spectrum with the chosen sigma-to-noise ratio. For each realization, parametrized by a given age and metallicity, we maximize the likelihood in the age and metallicity parameter space and determine the age marginalizing over the metallicity. The metallicity is allowed to change from 0.2 to $2.5 \mathrm{Z}_{\odot}$. The (rest frame) wavelength range used goes from 2500 to $7500 \AA$.

By comparing the long-dashed and the solid grey areas, which are the $1 \sigma$ region of the Monte-Carlo simulated errors, we see that the recovery is excellent and without bias. Even for a $S / N$ of only 10, the age-metallicity degeneracy is lifted when blue light from (rest frame) $2500 \AA$ is observed. 


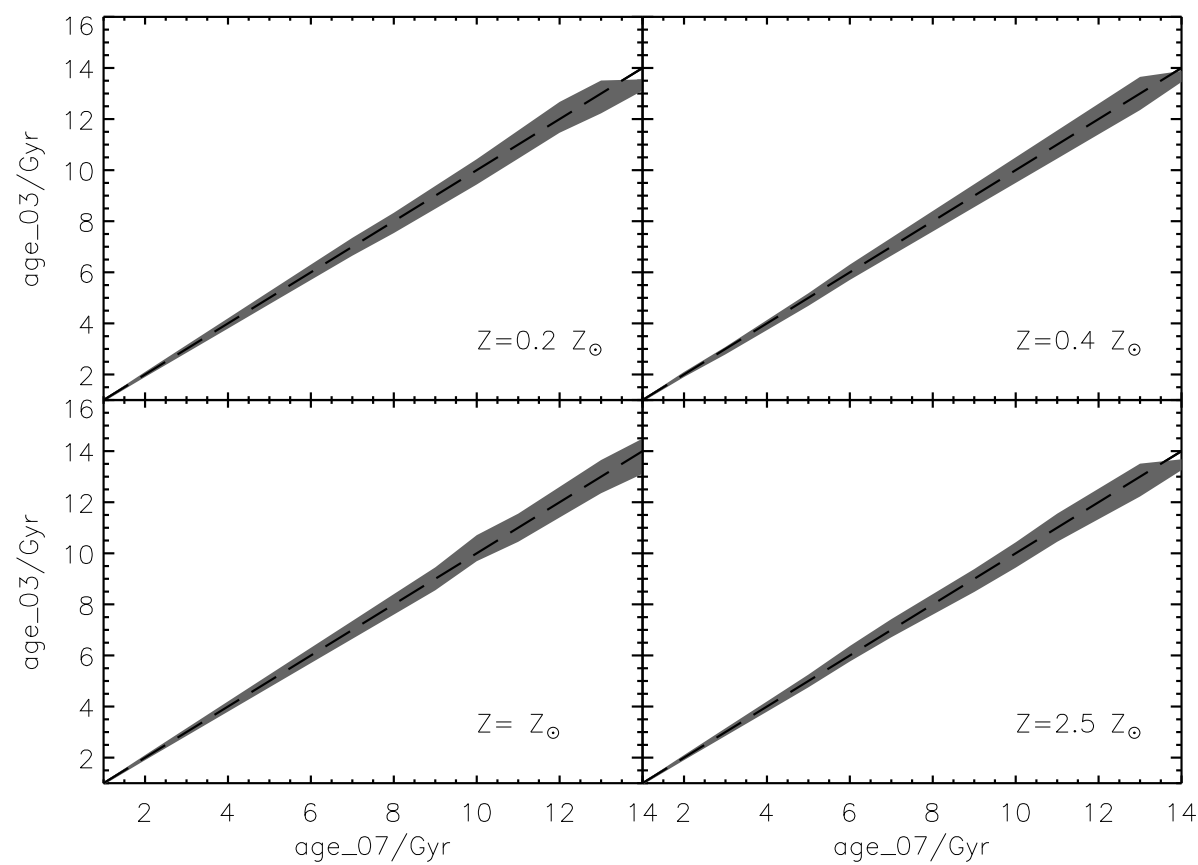

Figure 1. For sufficiently high $S / N$ data over sufficient wavelength range, the age-metallicity degeneracy is lifted. Input mock spectra are from the library by CB08 to which we have added random noise with $S / N=10$ per resolution element of $3 \AA$. The thin solid line is the input age. When we fit the same CB08 spectra to these mock spectra with both the age and metallicity as free parameters, we find a very good recovery of the age, with a random dispersion smaller than a few percent. The age-metallciity degeneracy is removed from spectra with blue-light coverage $(2500-7500 \AA)$ and modest $S / N$.

\section{Stellar-Population Uncertainties}

We next investigate the uncertainty that arises in the age determination from uncertainties in the stellar-population models. To do so, we take the mock spectra from $\$ 2$ with the CB08 models and then recover their age (marginalizing over the metallicity) with a different set of stellar-population models. Here, we use the Bruzual \& Charlot 2003 32] models which rely on a different set of stellar interior models (Geneva instead of Padova used by CB08) and different stellar atmosphere models. It should be noted that this exercise will generally over-estimate the uncertainty, because the models may have treatments of different phases of stellar evolution that are not up to date, or because some may be missing phases of stellar evolution 33. As models get updated, they will hopefully converge toward a more unified picture 34].

Figs. 23 show the comparison between the two different models for different metallicities and for different wavelength coverages. As in Fig. 1, the shaded grey regions correspond to the $1 \sigma$ region from 100 Monte-Carlo realizations. Fig. 2 uses a (rest frame) spectral range from 2500 to $7500 \AA$ while Fig. 3 drops the blue light and covers only the range from (rest frame) 4500 to $7500 \AA$. In both cases the $S / N$ is 20 


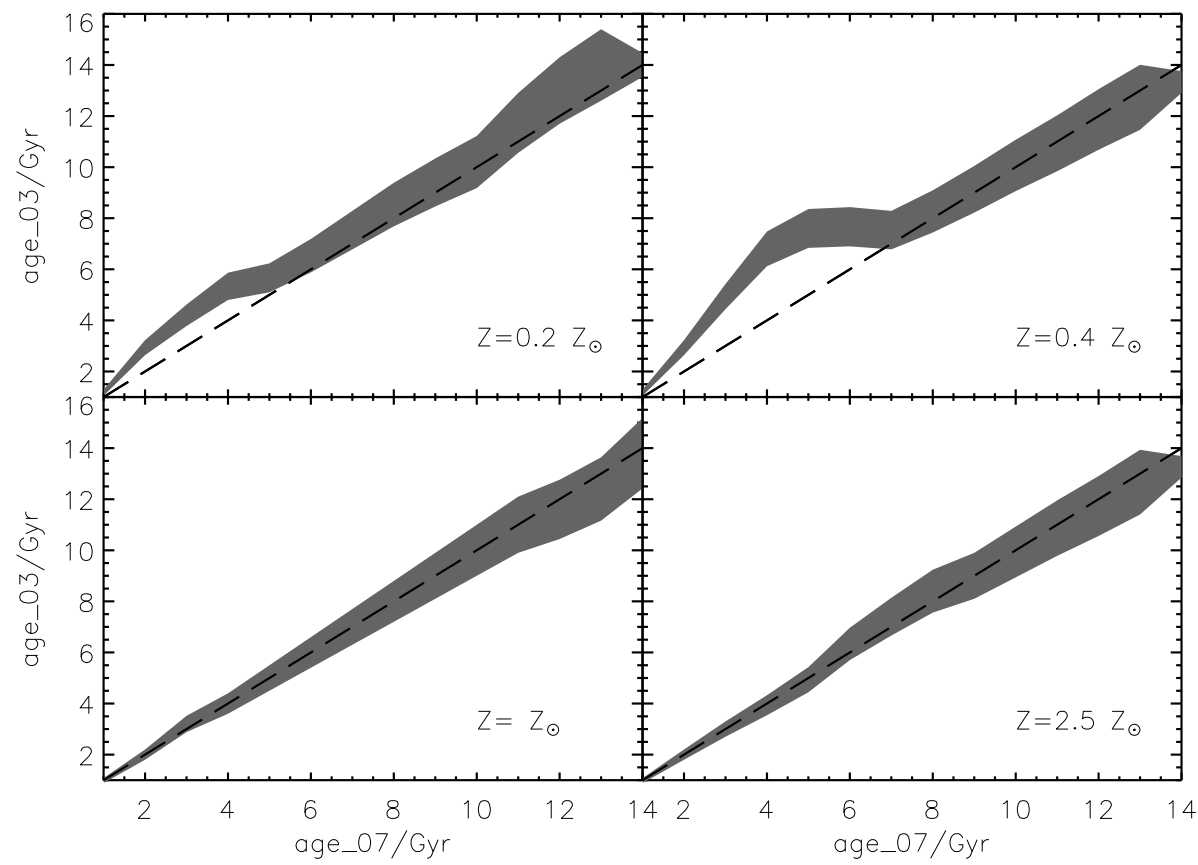

Figure 2. Age recovery for two different stellar-population models, CB08 and BC03, which rely on different input stellar physics (see text). The (rest frame) wavelength range of the fitted spectrum is 2500 to $7500 \AA$. Note the good age recovery despite different input physics, especially at higher metallicities where the absorption lines are stronger and help lift the age-metallicity degeneracy.

per resolution element of $3 \AA$. Inspection of Fig. 2 shows that there is good agreement in the age recovery for all ages and metallicities despite the different stellar input physics in the models. The recovery is especially good at higher metallicities, which corresponds to the typical metallicity of the old, massive galaxies found in clusters. This is expected because the stronger metallic absorption lines provide a better leverarm to determine the metallicity and thus break the age-metallicity relation. Note that the new CB08 models like Maraston [35] and SPEED [37, 36], apply a correction to properly model the giant branches.

\subsection{Importance of blue wavelength coverage}

It is apparent from comparison of Figs. 2 and 3 that the wavelength coverage of the observations is crucial, as the agreement degrades quickly with observations that do not include light bluewards of the $4000 \AA$ break. The blue end is particularly important, as line-blanketing at the blue end allows a more effective breaking of the degeneracy between the age and metallicity. It is this consideration that motivates the use of the Low Resolution Imaging Spectrometer (LRIS, [13]) on the Keck I telescope, which is senstive down to $\sim 3200 \AA$.

Because the method relies only on measuring relative ages, it is useful to illustrate the sensitivity of the observed spectrum to this parameter. In Fig. 4 we show the 


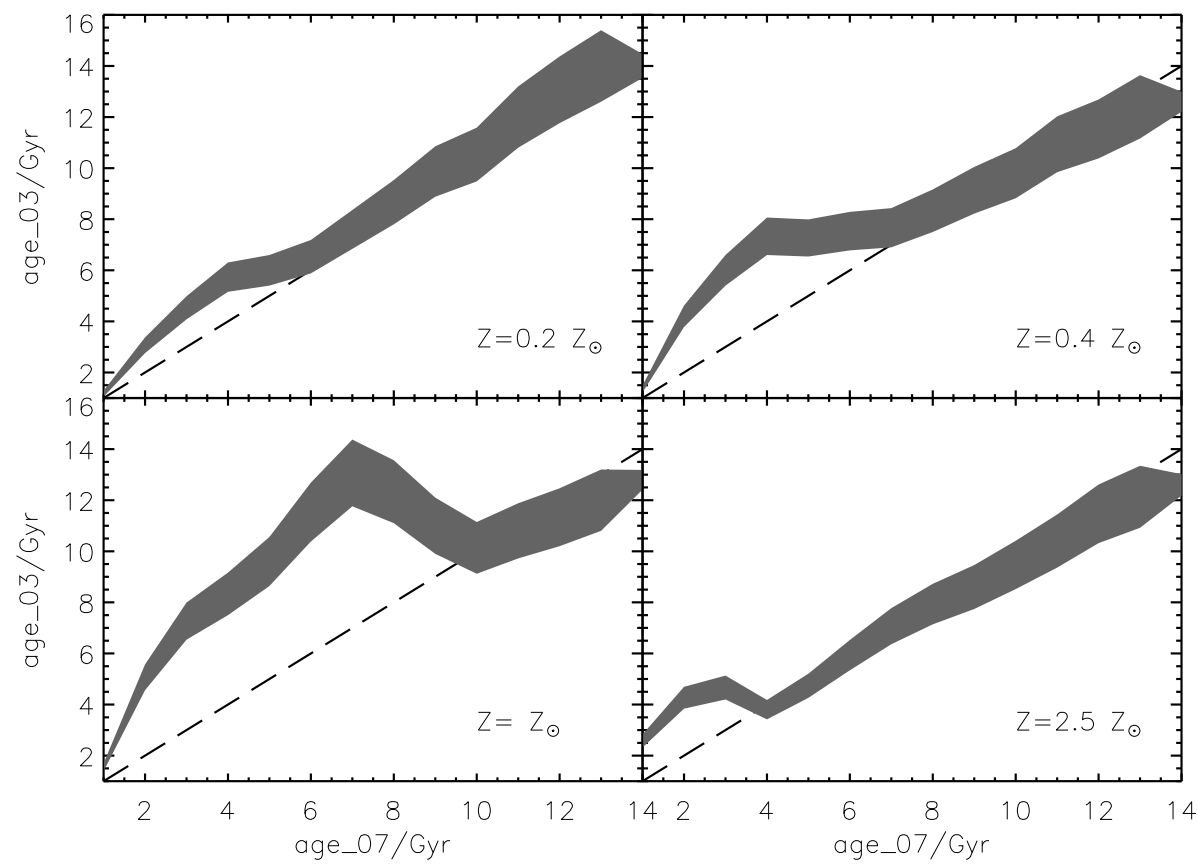

Figure 3. Same as Fig. 2 but for a (rest frame) wavelength range of 4500 to $7500 \AA$ A. Note that it becomes increasingly diffcult to recover the age when blue light in the spectrum is not measured.

relative flux of spectra for different ages with respect to a 2 Gyr old solar metallicity population. All spectra have been arbitrarily normalised to unity at $7500 \AA$. Even by eye the differences at a single wavelength are notable, above $10 \%$. The figure also illustrates how the greatest sensitivity comes from observations at the blue end of the spectra, $\approx 3000 \AA$.

\section{Galaxy Spectra Sample}

\subsection{Keck observations}

Motivated by the above considerations, we have started a program to obtain spectra of bright cluster galaxies with the Keck/LRIS instrument. We targeted rich galaxy clusters in order to obtain a sample as large as possible of red galaxies at redshift $z<1$. Most of the clusters are well-known, rich X-ray clusters from a variety of samples such as the Abell catalog [38, the ROSAT Cluster Survey (RCS; 39]) and the Massive Cluster Survey (MACS; 40]). In the redshift range $0.5<z<1$, fewer rich $\mathrm{X}$-ray clusters are known, so we targeted (and confirmed) two of the richest Spitzer mid-infrared selected cluster candidates from the IRAC Shallow Cluster Survey [41. Many of the targeted clusters were also observed in the near-infrared cluster survey of [42, which provided a valuable and consistent astrometric resource for slitmask designs. The list of clusters targeted is presented in Table 3.1 and an example of 


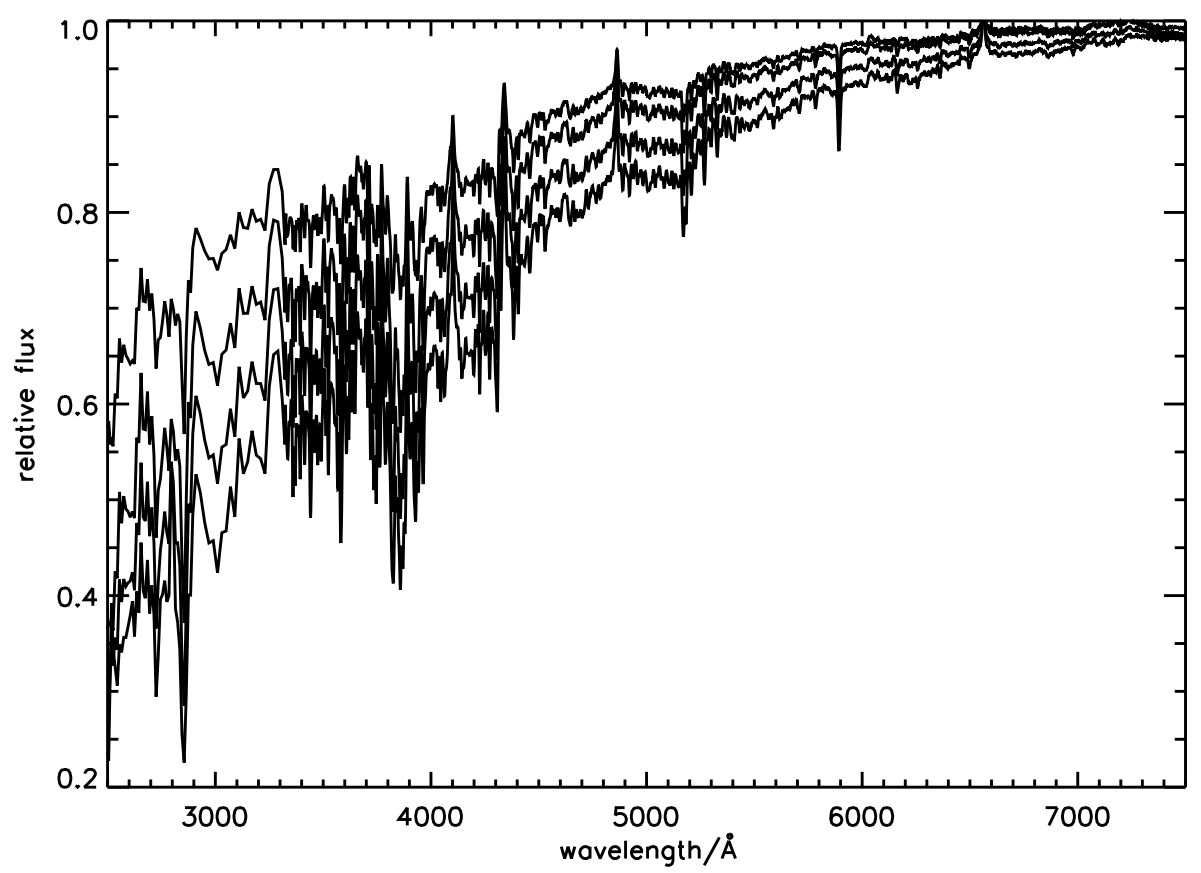

Figure 4. Relative flux of theoretical single stellar populations (SSPs) in the wavelength range 2500 to $7500 \AA$ for four different ages: 3, 5, 7,9 Gyr, from top to bottom as a ratio of the 2 Gyr spectrum. The plot illustrates how differential ages are measured and the essential role of blue wavelength coverage for this experiment.

observed spectra is shown in Fig. 5 ,

The key goal of this program is to provide high-signal-to-noise, wide-wavelengthcoverage spectroscopy of a large number of early-type galaxies at moderate redshifts. These spectra are then modeled to derive the ages of the galaxy stellar populations. Since rest-frame UV light probes the youngest and most massive stars in a galaxy, blue sensitivity is crucial for this experiment. Of all the optical spectrographs on $8-10 \mathrm{~m}$ class telescopes currently available, LRIS on the Keck I telescope is unique in being the only dual-beam spectrograph, thus providing sensitive observations across the entire optical window $(\lambda \sim 3200 \AA-1 \mu \mathrm{m})$.

We obtained the blue channel data with the 400-line $\mathrm{mm}^{-1}$ grism, which has a central wave-length of $3400 \AA$ and a spectral range of $4450 \AA$. We obtained the red channel data with the 400 -line $\mathrm{mm}^{-1}$ grating, which has a central wavelength of $8500 \AA$ and a spectral range of $3800 \AA$. Combining the blue- and red-channel data from the two dichroic settings, sources typically had final spectra which spanned the entire $\sim 3200 \AA-1 \mu \mathrm{m}$ optical window, albeit with higher noise at the short and long wavelength extremes. Based on analysis of sky lines, sources filling the 1.5" wide slitlets used for these observations have resolution $\lambda / \Delta \lambda \sim 500$ and $\sim 650$ for the blue and red channels, respectively. Standard stars from 43 were observed with the same instrument conguration for the purposes of spectrophotometric 
Table 1. Galaxy clusters observed.

\begin{tabular}{|c|c|c|c|c|}
\hline Galaxy Cluster & RA (J2000) & Dec (J2000) & $z$ & UT Date \\
\hline MS 0906.5+1110 & $09: 09: 12.7$ & $+10: 58: 29$ & 0.172 & 2005 Feb 10 \\
\hline MS $1253.9+0456$ & $12: 56: 00.0$ & $+04: 40: 00$ & 0.230 & 2008 Jul 1 \\
\hline Abell 1525 & $12: 21: 57.8$ & $-01: 08: 03$ & 0.260 & 2007 Dec 18 \\
\hline MS 1008.1-1224 & $10: 10: 32.3$ & $-12: 39: 52$ & 0.301 & 2007 Dec 17 \\
\hline CL 2244-0205 & $22: 47: 13.1$ & $-02: 05: 39$ & 0.330 & 2007 Dec $17-18$ \\
\hline Abell 370 & $02: 39: 53.8$ & $-01: 34: 24$ & 0.374 & 2007 Dec 18 \\
\hline MACS J1720.2+3536 & $17: 20: 12.0$ & $+35: 36: 00$ & 0.389 & 2008 Sep 3 \\
\hline CL $0024+16$ & $00: 26: 35.7$ & $+17: 09: 45$ & 0.394 & 2007 Dec 18 \\
\hline MACS J0429.6-0253 & 04:29:41.1 & $-02: 53: 33$ & 0.400 & 2008 Sep 3 \\
\hline MACS J0159.8-0849 & 01:59:00.0 & $-08: 49: 00$ & 0.405 & 2008 Sep 3 \\
\hline Abell 851 & $09: 43: 02.7$ & $+46: 58: 37$ & 0.405 & 2007 Dec 17 \\
\hline GHO $0303+1706$ & 03:06:19.1 & $+17: 18: 49$ & 0.423 & 2005 Feb 10 \\
\hline MS $1621.5+2640$ & $16: 23: 00.0$ & $+26: 33: 00$ & 0.428 & 2008 Jul 1 \\
\hline MACS J1610.6+3810 & $16: 21: 24.8$ & $+38: 10: 09$ & 0.465 & 2008 Sep 3 \\
\hline MACS J0257.1-2325 & 02:57:09.1 & $-23: 26: 06$ & 0.505 & 2008 Sep 3 \\
\hline MS 0451.6-0306 & $04: 54: 10.8$ & $-03: 00: 57$ & 0.539 & 2005 Feb 10 \\
\hline MS 0451.6-0306 & $04: 54: 10.8$ & $-03: 00: 57$ & 0.539 & 2007 Dec $17-18$ \\
\hline Boötes 10.1 & $14: 32: 06.0$ & $+34: 16: 47$ & 0.544 & 2008 Sep 3 \\
\hline CL $0016+16$ & $00: 18: 33.5$ & $+16: 25: 15$ & 0.545 & 2007 Dec 18 \\
\hline MACS J2129.4-0741 & $21: 26: 46.9$ & $-07: 54: 36$ & 0.570 & 2008 Jul 1 \\
\hline MACS J0025.4-1222 & $00: 25: 09.4$ & $-12: 22: 37$ & 0.578 & 2008 Jul 1 \\
\hline MACS J0647.7+7015 & $06: 47: 50.5$ & $+70: 14: 55$ & 0.591 & 2009 Mar 2 \\
\hline MACS J0647.7+7015 & $06: 47: 50.5$ & $+70: 14: 55$ & 0.591 & 2009 Mar 3 \\
\hline MACS J0744.8+3927 & $07: 44: 52.5$ & $+39: 27: 27$ & 0.697 & 2009 Mar 2 \\
\hline MACS J0744.8+3927 & $07: 44: 52.5$ & $+39: 27: 27$ & 0.697 & 2009 Mar 3 \\
\hline RCS $2318+0034$ & $23: 18: 31.5$ & $+00: 34: 18$ & 0.756 & 2008 Sep 3 \\
\hline MS 1054.5-0321 & $10: 56: 59.5$ & $-03: 37: 28$ & 0.828 & 2002 Mar 11 \\
\hline Boötes 10.8 & $14: 32: 06.0$ & $+34: 16: 47$ & 0.921 & 2008 Jul 1 \\
\hline
\end{tabular}

calibration. Observations were generally obtained with two dithered exposures per dichroic conguration with typical integration times of $900 \mathrm{~s}$ to $1800 \mathrm{~s}$, depending on the cluster redshift and observing conditions. This allowed both improved cosmic ray rejection and, by pair-wise subtraction of the images, removal of the fringing which strongly affects the long wavelength $(\lambda>7200 \AA)$ LRIS data. This required minimum slitlet lengths of approximately 10". Since LRIS has an atmospheric dispersion corrector, mask position angles were optimized based on the cluster orientation and no special attention was necessary to align the masks with the parallactic angle. We show in Fig. 5] an example of our observed spectra. See the companion paper [9] for more details of the observations, reductions, as well as a catalog of all $\approx 500$ redshifts measured by this project.

\subsection{SDSS, SPICES and VVDS spectra}

We have extended our Keck observations with the following datasets. We take advantage of the improvements in calibration provided by the SDSS spectroscopic 


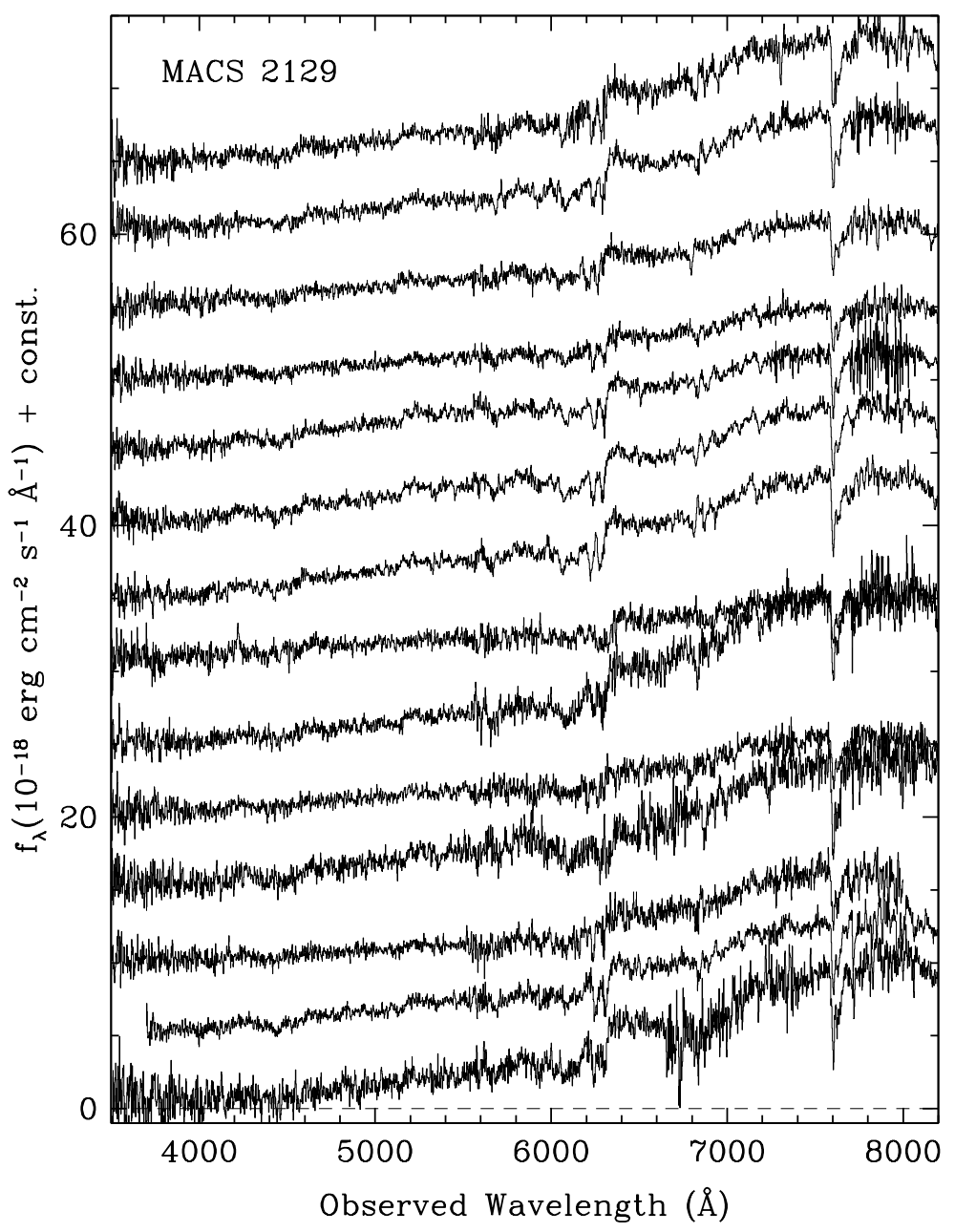

Figure 5. Example of spectra from our observing program. Observed Keck spectra of red galaxies in the cluster MACS J2129.4-0741 at $z=0.570$.

pipeline over our previous study [22, which was based on the early data release (EDR) sample, and we take advantage of the increased number of luminous red galaxies (LRGs) available in the public Data Release 6 (DR6) due to the larger survey area. In addition, we supplement our analysis using publicly available, high quality data from several surveys that targeted LRGs at higher redshift than the SDSS. The SPICES sample [4] targeted infrared-selected galaxies $(K<20)$ in about $1 / 3$ square degree of the sky. Most of the spectra were taken at the Keck telescope. The VVDS [45] is the VIMOS-VLT survey carried out by the VLT/ESO telescope that spectroscopically targeted galaxies in the range $17.5 \leq I_{\mathrm{AB}} \leq 24$. Neither of these surveys was aimed purely at LRGs, and so their efficiency at providing passively-evolving galaxies is significantly smaller than our Keck-survey project presented here. 


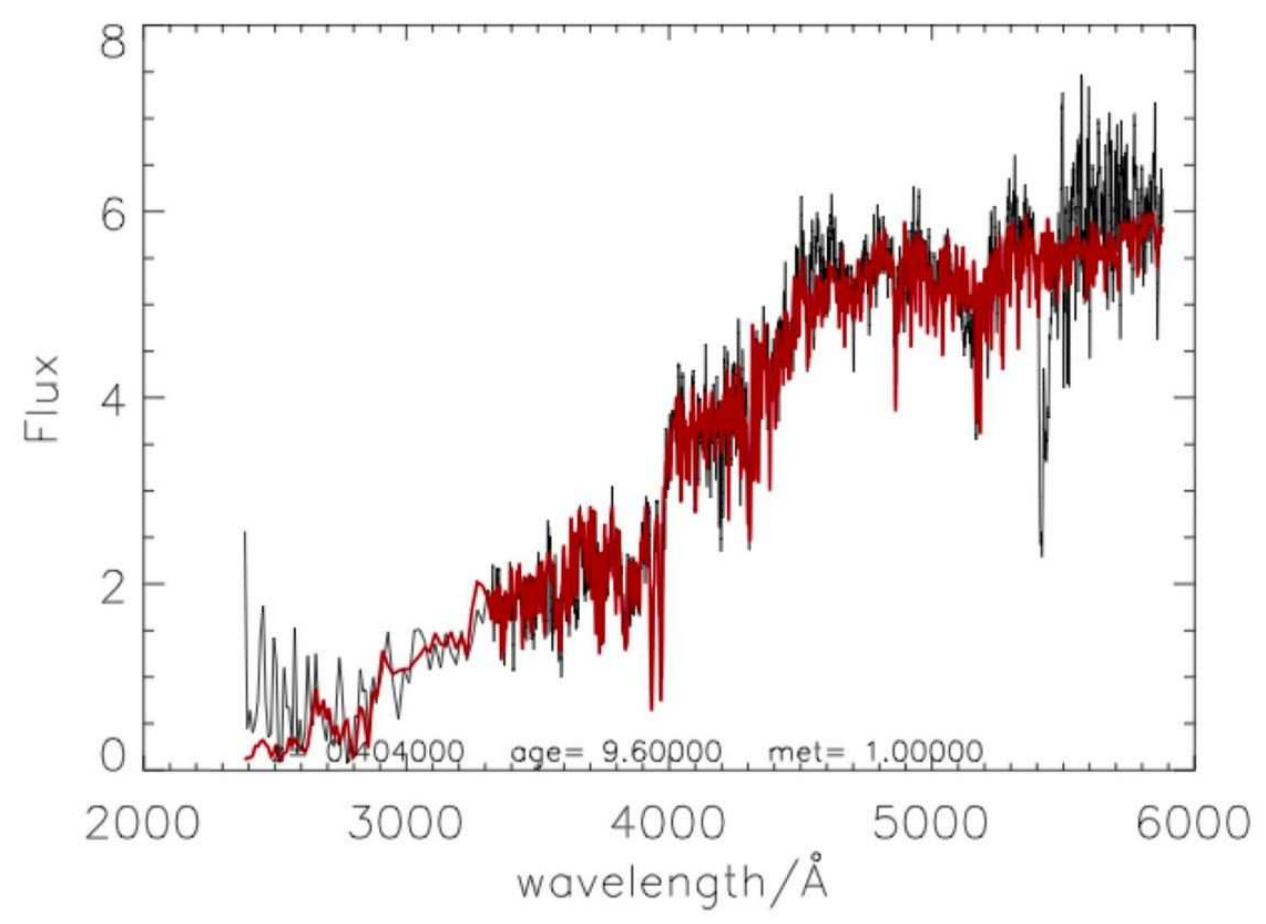

Figure 6. Example of a the model fit (red line) to observed spectra (black line) on the red envelope for a galaxy in the cluster MACS J0429.6-0253 at $z=0.400$. The best fit model has solar metallicity and an age of $9.6 \mathrm{Gyr}$.

\section{Measurement of the Age-Redshift Relation}

We now proceed to measure $d t / d z$ as a function of redshift. We fit single stellarpopulation spectra from the BC03 library for the full range of age and metallicity. We choose to use the BC03 library because it is the most widely used and eases the task of other researchers to reproduce our results. Fig. [6 shows an example of a model fit to an observed spectrum of a galaxy on the red envelope in the cluster MACS J0429.6-0253. However, significant improvements in modeling stellar populations have taken placed between the release of $\mathrm{BC} 03$ and now, with new stellar libraries, new models and new differential methods that have improved the fit of the models to the data. This is an active field where researchers are converging to a "standard" model; until such a model is uncovered, the standard remains BC03. However, as we show below, the choice of stellar population model does not change the differential age measurements for the red envelope. We generate single stellar population (SSP) models with BC03 for ages of $0.1-15 \mathrm{Gyr}$ and metallicities of $0.1-2.5 Z_{\odot}$. To obtain the best age, we perform a $\chi^{2}$ fit and marginalize over the metallicity to obtain error bars. Star formation is assumed to occur in a single burst of duration much shorter $(<5 \%)$ than the current age of the galaxy. We discard fits that have a reduced- $\chi^{2}$ value $>1.2$, as experience has shown that these galaxies have data issues (e.g., poor $S / N$ ratio due to a bright moon and/or poor weather, poor sky subtraction, contamination from nearby - and, in four cases, lensed - sources, contamination from an active nucleus), or are better 


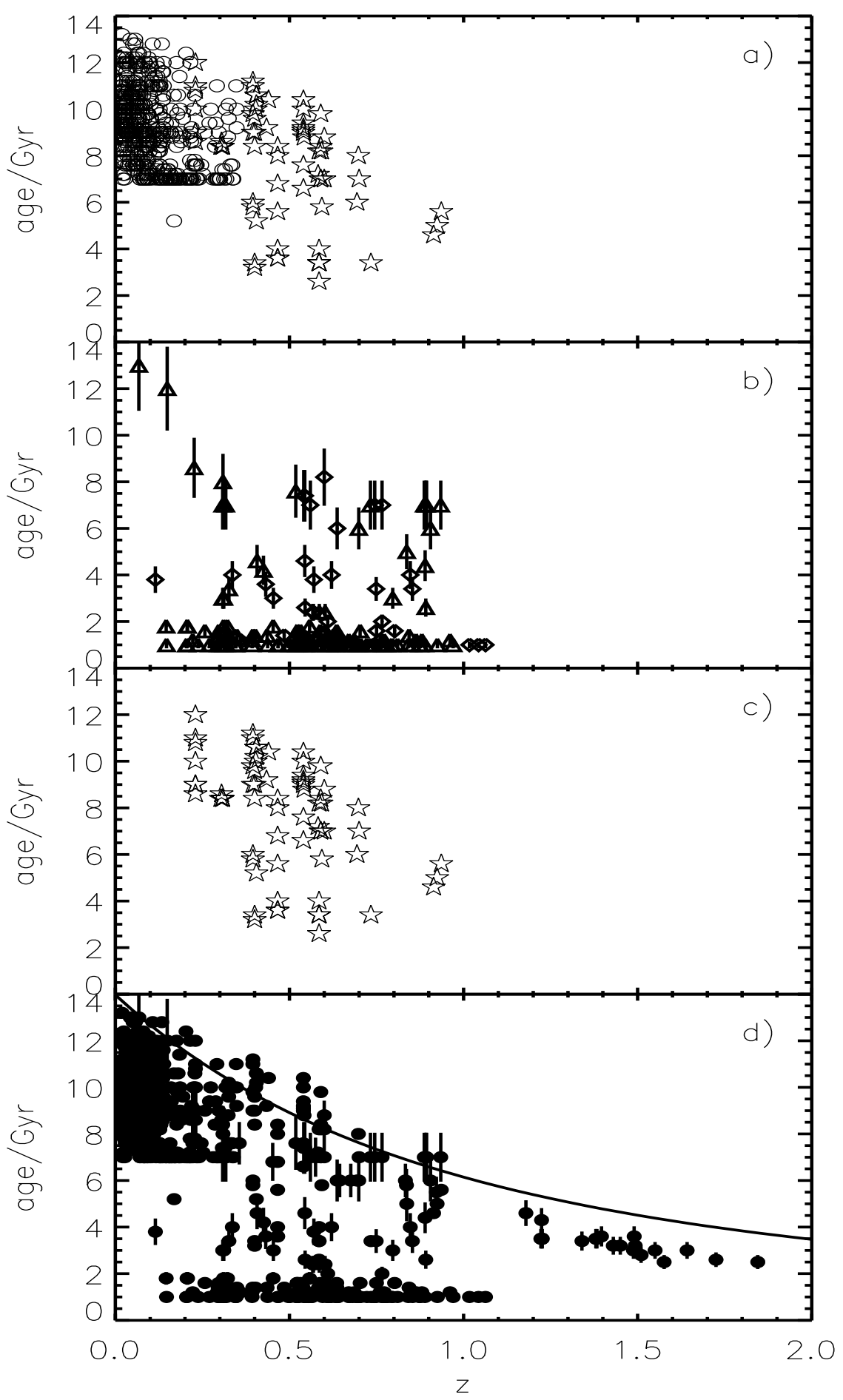

Figure 7. Age-redshift relation for (a) SDSS-LRG galaxies and Keck clusters; (b) SPICES (diamonds) and VVDS (triangles) galaxies; (c) Keck clusters; and (d) all samples, including the ones in Ref. 23]. The determination of $H(z)$ depends only on the determination of the upper (red) envelope; younger galaxies do not play any role. Note that there is a clear age-redshift relation for all samples. The solid line is the theoretical age redshift relation for the $\Lambda \mathrm{CDM}$ model. 


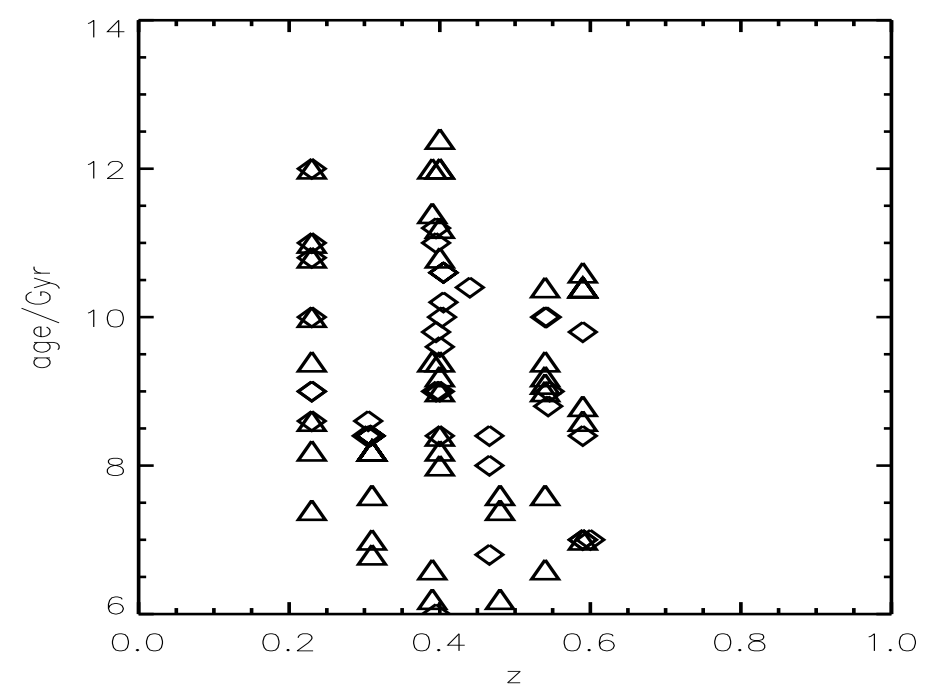

Figure 8. Ages obtained with the BC03 models (diamonds) and the CB08 models (triangles). The plot shows that the red-envelope in the age-redshift relation is not compromised by the choice of stellar population model. The solid line is the theoretical age redshift relation for the $\Lambda$ CDM model.

fit by an extended star-formation history and therefore do not belong to the category of passively evolving stellar populations. We also allow for dust in the fit following Ref. [30] and discard those galaxies that contain dust in their fits; i.e., those where the dust parameter used in Ref. [30] is nonzero. To compute the differential ages, we use the solar metallicity, 5 Gyr-old model as our reference and compute $d t$ with respect to it.

The results of the age determination are presented in Fig. 7, where the four panels show the age-redshift relation for SDSS-LRG galaxies and Keck clusters (upper panel), SPICES (diamonds) and VVDS (triangles) galaxies (middle-upper panel), just Keck clusters (middle-lower panel), and all samples including the ones in Ref. 23] (bottom panel). Typical errors on the age are shown for in the middle-upper panel to avoid cluttering the plots. We also show in Fig. 8 a comparison of the age-redshift relation for the Keck cluster sample using BC03 (triangles) and CB08 (diamonds) models. Besides two discrepant galaxies, which in any case are consistent within the errors with the red envelope edge, the defined edge is nearly identical. This illustrates that the edge does not overly depend on the synthetic stellar population model.

Note that there is a clear age-redshift relation for all samples, either taken individually or together. For comparison, the solid line shows the theoretical age redshift relation for the standard $\Lambda \mathrm{CDM}$ model. Also note the excellent performance of the clusters observed with Keck at selecting the oldest stellar populations, effectively from just two nights of data in good conditions. The ages derived from the Keck clusters nicely fill in the gap in the redshift range $0.3<z<1.0$. Finally, we note that the different data samples are consistent with each other.

We are interested in computing the "edge" of this distribution in the age-redshift plane. As demonstrated in Ref. [46], this is most efficiently done by computing where 


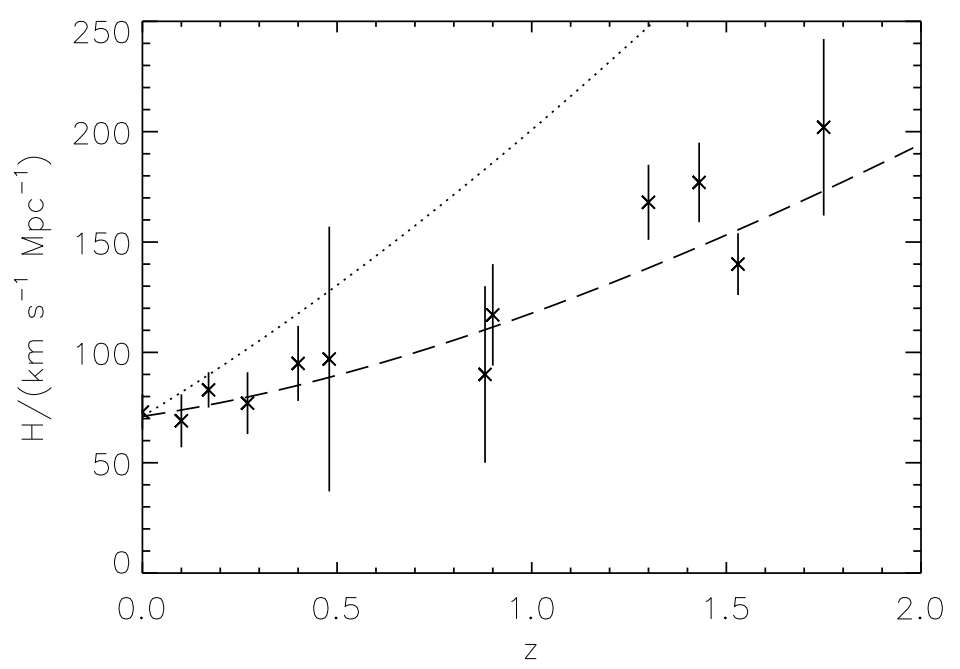

Figure 9. The $H(z)$ measurement from our sample of passively evolving galaxies. The points at $z>1$ are taken from Ref. 23 , while the point at $z=0$ is from the Hubble Key Project [5]. The dashed line is the $\Lambda$ CDM model while the dotted line is an Einstein-de-Sitter model.

the distribution, as fitted by a one-sided Gaussian, drops by $50 \%$. The new data are all at $z<1$. While the recovered age-redshift relation is in good agreement with the SDSS one at $z<0.3$, the few additional data points do not change the determination used in Ref. 23. Thus, we concentrate here on the redshift range $0.35<z<1.0$. We sub-divide it in two intervals: $0.35<z<0.6$ and $0.6<z<1.0$. The second interval is wider because it is populated by fewer galaxies. Each of these intervals is divided in two equal bins where the edge of the age-redshift relation is found in a way similar to that described in Ref. [46, and we also determine $H(z)$ following Ref. [46]. We have checked that the $H(z)$ determination is robust, within the error bars, to the choice of binning and the choice of galaxies that populate the edge.

\section{Constraints on Cosmological Parameters}

We now proceed to place constraints on cosmological parameters using the above determination of $H(z)$ in combination with the latest results from the WMAP 5-year release 48. For some applications we also consider the latest determination of $H_{0}$ from Ref. 47. The $H(z)$ data help constrain cosmological parameters beyond the standard $\Lambda \mathrm{CDM}$ model, in particular for parameters that affect the Universe expansion history or that have degeneracies with expansion-history parameters. We find improved constraints for models with arbitrary curvature and dark-energy equation-of-state parameter $w \neq-1$, and for models with non-standard neutrino properties.

We have obtained Monte Carlo Markov chains for the WMAP 5-year data 48, 49, from the LAMBDA web site (www.lambda.gsfc.gov) and we importance-sampled them with the $H(z)$ data. For the non-flat dark-energy models we run independent Monte Carlo Markov chains. Figs.10 16illustrate how the $H(z)$ data help to constrain 
Table 2. $H(z)$ from passively evolving galaxies.

\begin{tabular}{cc}
\hline & $H(z)$ \\
$z$ & {$\left[\mathrm{~km} \mathrm{sec}^{-1} \mathrm{Mpc}^{-1}\right]$} \\
\hline 0 & $73 \pm 8$ \\
0.1 & $69 \pm 12$ \\
0.17 & $83 \pm 8$ \\
0.27 & $77 \pm 14$ \\
0.4 & $95 \pm 17$ \\
0.48 & $97 \pm 60$ \\
0.88 & $90 \pm 40$ \\
0.9 & $117 \pm 23$ \\
1.3 & $168 \pm 17$ \\
1.43 & $177 \pm 18$ \\
1.53 & $140 \pm 14$ \\
1.75 & $202 \pm 40$ \\
\hline
\end{tabular}

cosmological parameters beyond the standard model.

We start by considering constraints on dark energy where the dark-energy equation of state is parameterized as $w(z)=w+w_{a}(1-a)$ and where we allow for arbitrary curvature; we call this model the open-w(z)CDM model. Fig. 10] shows the improvement in the $w, w_{a}$ plane over $\mathrm{CMB}+H_{0}$ constraints where the $H_{0}$ constraint comes from the Hubble Key Project [5. Fig. 11] shows constraints in the $w-\Omega_{\Lambda}$ plane, and constraints on curvature for the above parameterization of the equation of state are shown in Fig. 12. Adding the $H_{0}$ determination of Ref. [4] visibly improves the constraints only in Fig. 11, shown as the darker filled contours.

If we impose an equation-of-state parameter constant in redshift, then the constraints on the curvature improve noticeably, as seen in Fig. 13. The wide transparent contours are from WMAP5 data alone (with a top hat prior on $H_{0}<$ $100 \mathrm{~km} \mathrm{~s}^{-1} \mathrm{Mpc}^{-1}$ ); the smaller transparent contours are for WMAP5 data and the Ref. 47] $H_{0}$ determination; filled contours also add $H(z)$ data.

We next turn our attention to the constraints obtained on neutrino properties. Figs. 14 and 15] show the constraints that can be obtained on the number of relativistic species, $N_{\text {rel }}$. There is a noticeable improvement over previous constraints. We find $N_{\text {rel }}=4 \pm 0.5$ at the $1 \sigma$ level. Finally, we present constraints on the total mass of relativistic species. As shown in Fig. 16, the best constraints we obtain using the $H(z)$ data is $m_{\nu}<0.2 \mathrm{eV}$, which is an improvement of $\sim 20 \%$ over the constraints obtained using the new $H_{0}$ measurements by Ref. 47] and a factor of two improvement over our previous study [50].

Finally, we consider dark energy that is described by a slowly rolling scalar field and we attempt a parametric reconstruction of its potential $V(z)$. For this purpose we assume the Universe to be spatially flat and we follow the steps outlined in Ref. 23]. We approximate a generic potential as an expansion in Chebyshev polynomials and we truncate the expansion at second order (i.e., we have three dark-energy parameters). The quantity $[H(z)]^{2}$ is a suitably weighted integral in redshift of $V(z)$ (see Ref. [23; Eq. (29)). We then use the $H(z)$ data presented here, the $H_{0}$ determination of Ref. [47], and a weak $\Omega_{m}$ prior $\left(\Omega_{m}=0.27 \pm 0.070\right.$. The resulting reconstructed 


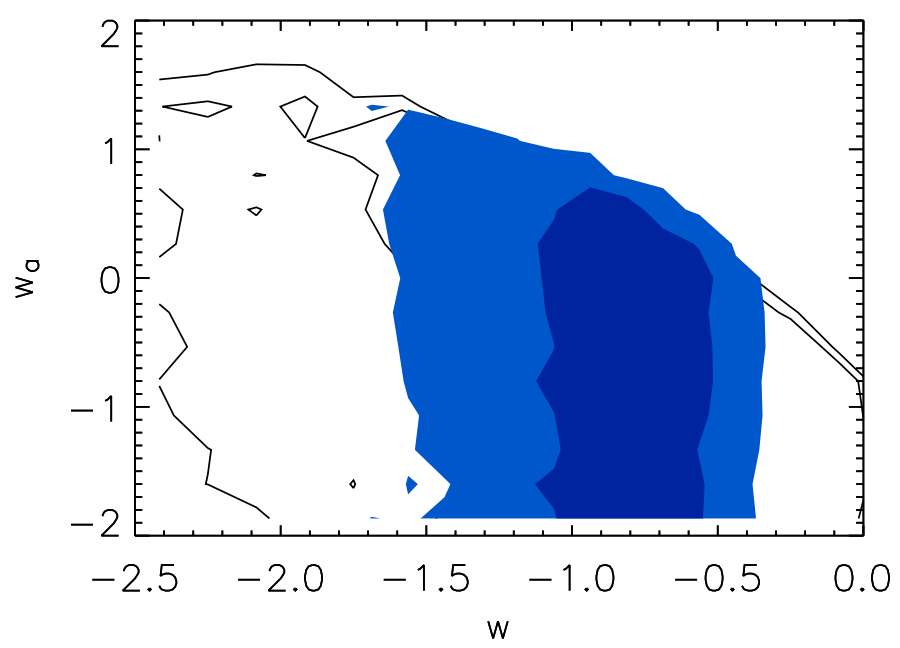

Figure 10. Constraints in the $w-w_{a}$ plane for an open-w(z)CDM where $w(z)=$ $w+w_{a}(1-a)$. The solid empty contours are for a model using WMAP5 and $H_{0}$ constraints. The filled contours use $H(z)$ information.

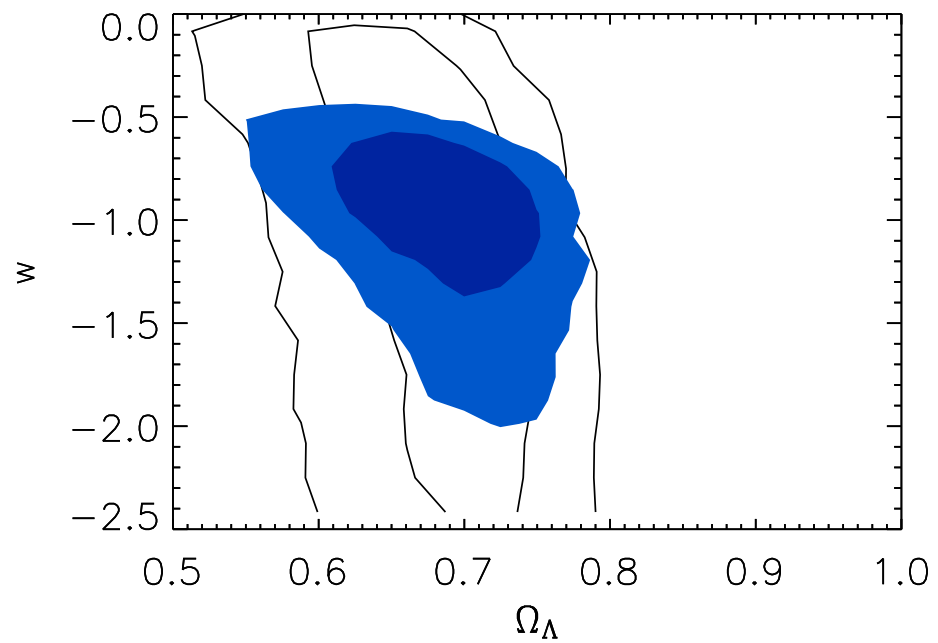

Figure 11. Constraints in the $w-\Omega_{\Lambda}$ plane for an open-w(z)CDM model. Solid contours are from the WMAP5 and $H_{0}$ datasets, while the solid contours use $H(z)$ information. 


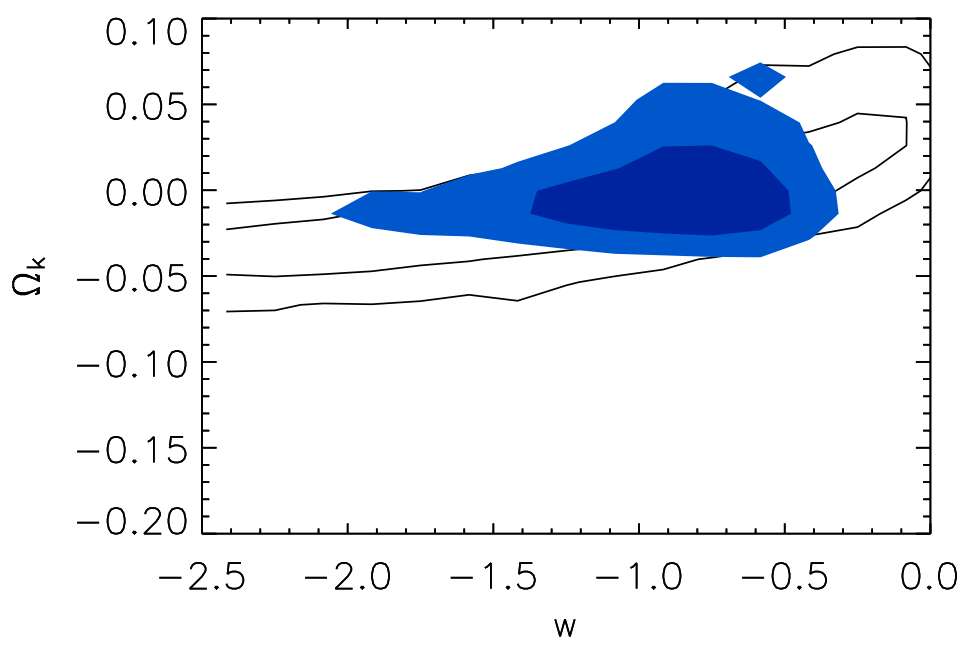

Figure 12. Constraints in the $w-\Omega_{k}$ plane for an open-w(z)CDM model. Solid contours are from the WMAP5 and $H_{0}$ (HST key project; [5) datasets, while the solid contours use $H(z)$ information.

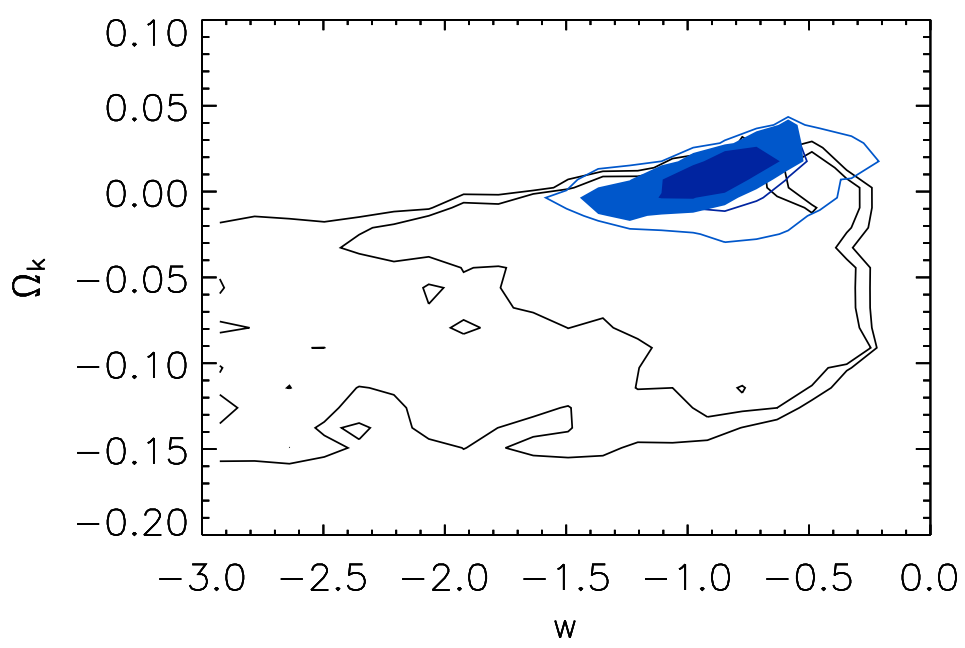

Figure 13. Constraints on $w$, assuming it constant, for an open-wCDM model. The solid empty contours are obtained using WMAP5 data; the solid blue contours add the $H_{0}$ constraint from Ref. [47; the filled contours also include $H(z)$ information. 


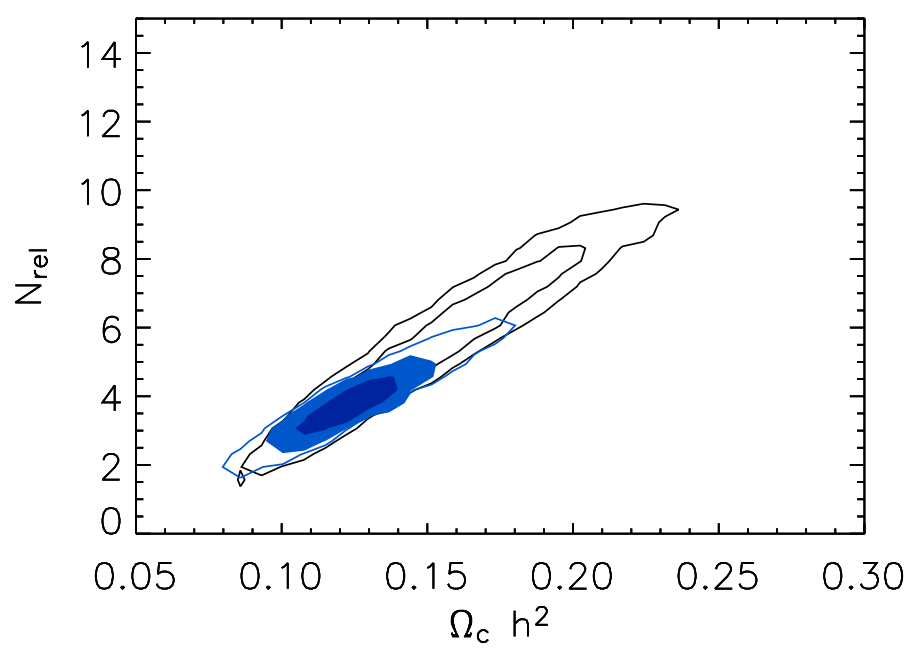

Figure 14. Constraints on the number of relativistic species. Solid black contours are with WMAP5 data only; blue transparent contours are for WMAP5 data and $H_{0}$; and blue filled contours are WMAP $5+H_{0}$.

$\mathrm{t}$

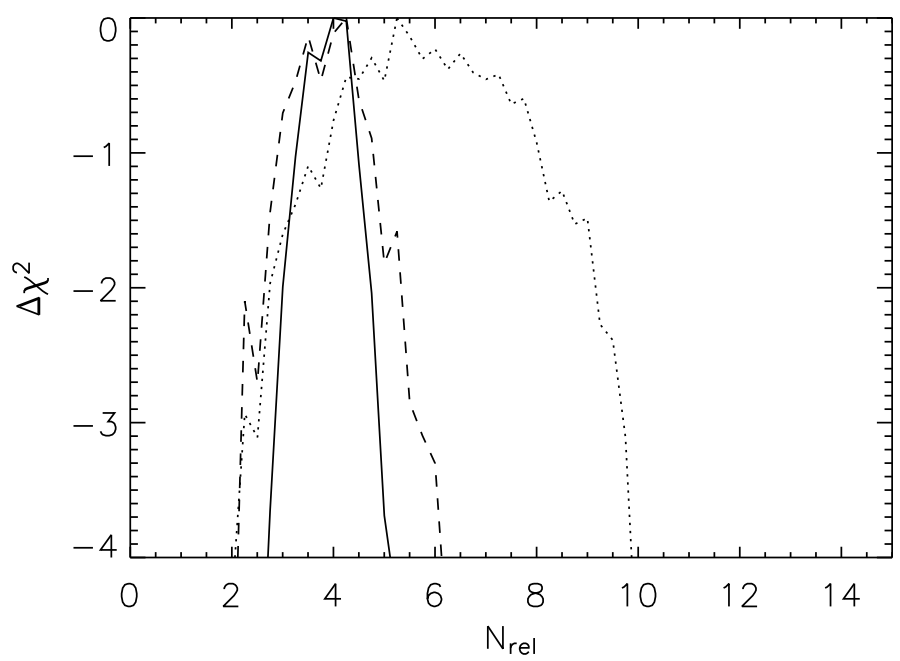

Figure 15. Constraint on the number of relativistic species using WMAP5 (dotted); WMAP5 with the $H_{0}$ measurement of Ref. 47] (dashed); and WMAP $5+H_{0}+H(z)$ data (solid line). 


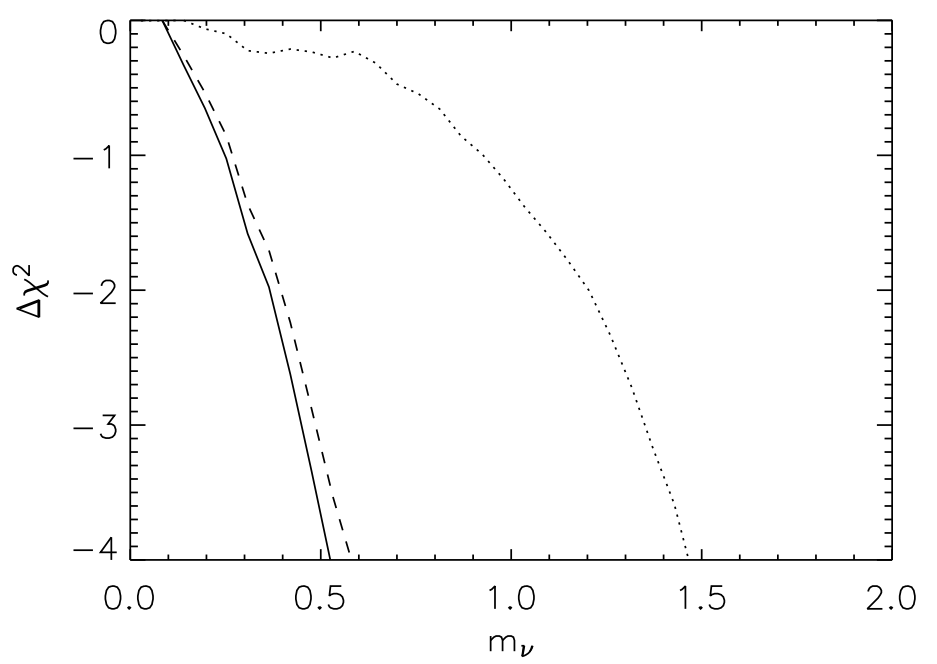

Figure 16. Constraints on the total mass of relativistic species, in $\mathrm{eV}$, using the WMAP5 data only (dotted line); including the new $H_{0}$ measurement from Ref. 47. (dashed line); and adding the $H(z)$ data which improves the $H_{0}$ constraints by $\sim 20 \%$. The total mass of relativistic species is constraint to be $<0.2 \mathrm{eV}$ at $1 \sigma$.

$V(z)$ is shown in Fig. 17. The reconstruction is fully consistent with a cosmological constant.

\section{Conclusions}

We report on a new measurement of the expansion history obtained from the ages of passively-evolving galaxies in galaxy clusters at $z<1.0$. Such observations provide a promising new cosmological constraint, particularly for understanding the evolution of the dark-energy density, with a relatively modest allocation of telescope time, effectively just two good nights at Keck. The current measurements already provide valuable constraints, and the success of this first campaign should motivate further measurements along these lines as well as a more intensive investigation of the theoretical underpinnings of the calculations and the associated uncertainties. Further, there has been significant advancement in the last few years in modeling stellar populations of LRG galaxies [52, 34, 51 and the differential technique has been recently applied very successfully to determine the metallicity of LRGs [53]. It may be that with additional effort on both the theoretical and observational side that the differential-age technique may ultimately provide an important new darkenergy avenue which complements supernova searches, weak lensing, baryon acoustic oscillations, and cluster abundances. 


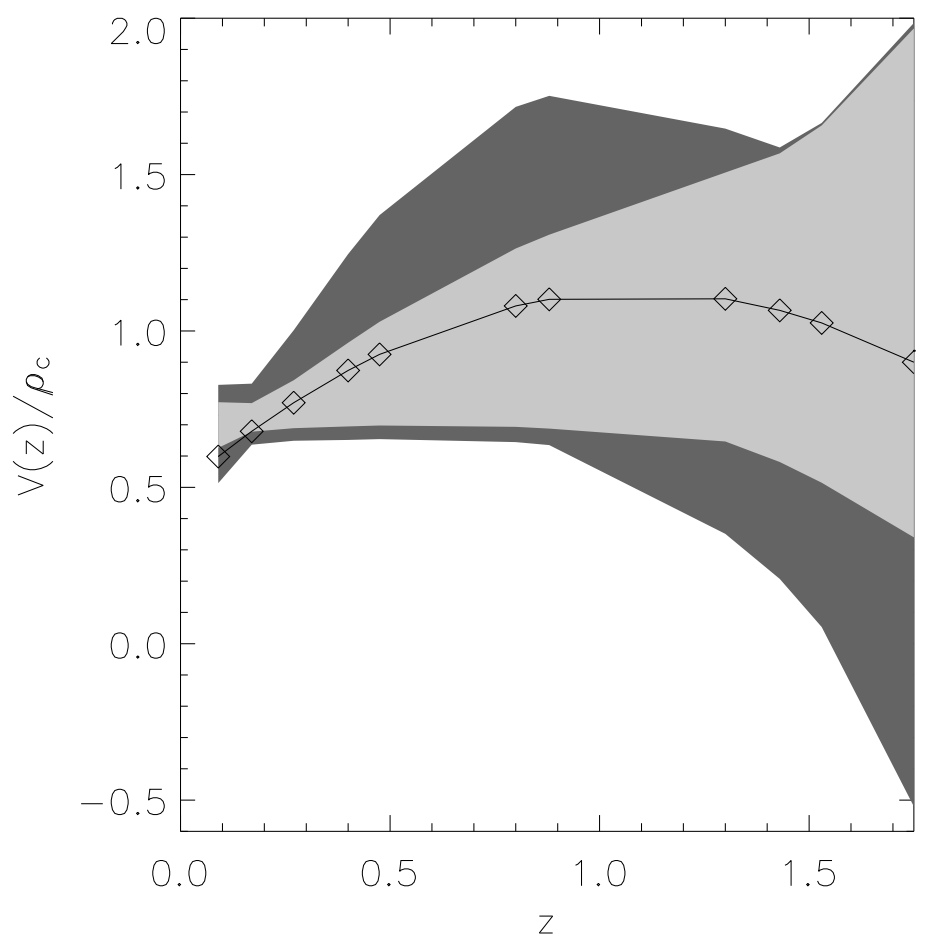

Figure 17. Reconstructed dark-energy potential $V(z)$ using an expansion in Chebyshev polynomials truncated at second order (i.e., three coefficients). To produce these constraints, we have used the $H(z)$ data presented here, the $H_{0}$ determination of Ref. [47, and a weak $\Omega_{m}$ prior $\left(\Omega_{m}=0.27 \pm 0.07\right)$. Gray regions show the $1 \sigma$ and $2 \sigma$ confidence regions; the solid line is the best fit $V(z)$. The diamonds show the redshifts at which $H(z)$ has been measured.

\section{Acknowledgments}

The work of DS was carried out at the Jet Propulsion Laboratory, operated by the California Institute of Technology under a contract with NASA. The work of RJ and LV is supported by funds from the Spanish Ministry for Science and Innovation AYA 2008-03531 and the European Union (FP7 PEOPLE-2002IRG4-4-IRG\#202182). MK was supported by DoE DE-FG03-92-ER40701 and the Gordon and Betty Moore Foundation. We thank the members of the SPICES and VVDS teams for making their spectroscopic data publicly available, and we acknowledge the use of the Legacy Archive for Microwave Background Data Analysis (LAMBDA). Support for LAMBDA is provided by the NASA Office of Space Science. Finally, the authors wish to recognize and acknowledge the very significant role and reverence that the summit of Mauna Kea has always had within the indigenous Hawaiian community. We are most fortunate to have the opportunity to conduct observations from this mountain. 


\section{References}

[1] S. Perlmutter et al. [Supernova Cosmology Project Collaboration], "Measurements of Omega and Lambda from 42 High-Redshift Supernovae," Astrophys. J. 517 (1999) 565 arXiv:astro-ph/9812133; A. G. Riess et al. [Supernova Search Team Collaboration], "Observational Evidence from Supernovae for an Accelerating Universe and a Cosmological Constant," Astron. J. 116 (1998) 1009 arXiv:astro-ph/9805201.

[2] G. Jungman, M. Kamionkowski, A. Kosowsky and D. N. Spergel, "Cosmological parameter determination with microwave background maps," Phys. Rev. D 54 (1996) 1332 arXiv:astro-ph/9512139; G. Jungman, M. Kamionkowski, A. Kosowsky and D. N. Spergel, "Weighing the universe with the cosmic microwave background," Phys. Rev. Lett. 76 (1996) 1007 arXiv:astro-ph/9507080; P. de Bernardis et al. [Boomerang Collaboration], "A Flat Universe from High-Resolution Maps of the Cosmic Microwave Background Radiation," Nature 404 (2000) 955 arXiv:astro-ph/0004404; A. D. Miller et al., "A Measurement of the Angular Power Spectrum of the CMB from l = 100 to 400," Astrophys. J. 524 (1999) L1 arXiv:astro-ph/9906421; S. Hanany et al., "MAXIMA-1: A Measurement of the Cosmic Microwave Background Anisotropy on angular scales of 10 arcminutes to 5 degrees," Astrophys. J. 545 (2000) L5 arXiv:astro-ph/0005123; A. H. Jaffe et al. [Boomerang Collaboration], "Cosmology from Maxima-1, Boomerang and COBE/DMR CMB Observations," Phys. Rev. Lett. 86 (2001) 3475 arXiv:astro-ph/0007333; N. W. Halverson et al., "DASI First Results: A Measurement of the Cosmic Microwave Background Angular Power Spectrum," Astrophys. J. 568 (2002) 38 arXiv:astro-ph/0104489]; B. S. Mason et $a l$. , "The Anisotropy of the Microwave Background to l = 3500: Deep Field Observations with the Cosmic Background Imager," Astrophys. J. 591 (2003) 540 arXiv:astro-ph/0205384]; A. Benoit et al. [the Archeops Collaboration], "Cosmological constraints from Archeops," Astron. Astrophys. 399 (2003) L25 arXiv:astro-ph/0210306]; J. H. Goldstein et al., "Estimates of Cosmological Parameters Using the CMB Angular Power Spectrum of ACBAR," Astrophys. J. 599 (2003) 773 arXiv:astro-ph/0212517; D. N. Spergel et al. [WMAP Collaboration], "First Year Wilkinson Microwave Anisotropy Probe (WMAP) Observations: Determination of Cosmological Parameters," Astrophys. J. Suppl. 148 (2003) 175 arXiv:astro-ph/0302209; C. L. Reichardt et al., "High resolution CMB power spectrum from the complete ACBAR data set," Astrophys. J. 694 (2009) 1200 arXiv:0801.1491 [astro-ph]]; C. L. Reichardt et al., "High resolution CMB power spectrum from the complete ACBAR data set," arXiv:0801.1491 [astro-ph]; D. N. Spergel et al. [WMAP Collaboration], "Wilkinson Microwave Anisotropy Probe (WMAP) three year results: Implications for cosmology," Astrophys. J. Suppl. 170 (2007) 377 arXiv:astro-ph/0603449; J. Dunkley et al. [WMAP Collaboration], "Five-Year Wilkinson Microwave Anisotropy Probe (WMAP) Observations: Likelihoods and Parameters from the WMAP data," Astrophys. J. Suppl. 180 (2009) 306 arXiv:0803.0586 [astro-ph]].

[3] W. J. Percival et al. [The 2dFGRS Collaboration], "The 2dF Galaxy Redshift Survey: The power spectrum and the matter content of the universe," Mon. Not. Roy. Astron. Soc. 327 (2001) 1297 arXiv:astro-ph/0105252.

[4] S. Dodelson et al. [SDSS Collaboration], "The three-dimensional power spectrum from angular clustering of galaxies in early SDSS data," Astrophys. J. 572 (2001) 140 arXiv:astro-ph/0107421.

[5] W. L. Freedman et al. [HST Collaboration], "Final Results from the Hubble Space Telescope Key Project to Measure the Hubble Constant," Astrophys. J. 553 (2001) 47 arXiv:astro-ph/0012376.

[6] P. J. E. Peebles and B. Ratra, "The cosmological constant and dark energy," Rev. Mod. Phys. 75, 559 (2003) arXiv:astro-ph/0207347]; T. Padmanabhan, "Cosmological constant: The weight of the vacuum," Phys. Rept. 380 (2003) 235 arXiv:hep-th/0212290]; E. J. Copeland, M. Sami and S. Tsujikawa, "Dynamics of dark energy," Int. J. Mod. Phys. D 15 (2006) 1753 arXiv:hep-th/0603057; J. Frieman, M. Turner and D. Huterer, "Dark Energy and the Accelerating Universe," Ann. Rev. Astron. Astrophys. 46 (2008) 385 arXiv:0803.0982 [astro-ph]]; E. V. Linder, "Mapping the Cosmological Expansion," Rept. Prog. Phys. 71 (2008) 056901 arXiv:0801.2968 [astro-ph]]; R. R. Caldwell and M. Kamionkowski, "The Physics of Cosmic Acceleration," arXiv:0903.0866 [astro-ph.CO];

[7] B. Ratra and P. J. E. Peebles, "Cosmological Consequences of a Rolling Homogeneous Scalar Field," Phys. Rev. D 37 (1988) 3406; R. R. Caldwell, R. Dave and P. J. Steinhardt, "Cosmological Imprint of an Energy Component with General Equation-of-State," Phys. Rev. Lett. 80, 1582 (1998) arXiv:astro-ph/9708069. 
[8] J. A. Peacock, P. Schneider, G. Efstathiou, J. R. Ellis, B. Leibundgut, S. J. Lilly and Y. Mellier, "Report by the ESA-ESO Working Group on Fundamental Cosmology," arXiv:astro-ph/0610906

[9] D. Stern, R. Jimenez, L. Verde, S. A. Stanford and M. Kamionkowski, "Cosmic Chronometers: Constraining the Equation of State of Dark Energy. II. A Spectroscopic Catalog of Red Galaxies in Galaxy Clusters," Astrophys. J. Suppl., submitted [arXiv:astro-ph/0907.3152].

[10] A. Albrecht et al., "Report of the Dark Energy Task Force," arXiv:astro-ph/0609591

[11] W. J. Percival, S. Cole, D. J. Eisenstein, R. C. Nichol, J. A. Peacock, A. C. Pope and A. S. Szalay, "Measuring the Baryon Acoustic Oscillation scale using the SDSS and 2dFGRS," Mon. Not. Roy. Astron. Soc. 381 (2007) 1053 arXiv:0705.3323 [astro-ph]].

[12] D. J. Eisenstein et al. [SDSS Collaboration], "Detection of the Baryon Acoustic Peak in the Large-Scale Correlation Function of SDSS Luminous Red Galaxies," Astrophys. J. 633 (2005) 560 arXiv:astro-ph/0501171.

[13] J. B. Oke et al., "The Keck Low Resolution Imaging Spectrometer," Publ. Astrophys. Soc. Pac. 107 (1995) 375

[14] H. J. Seo and D. J. Eisenstein, "Probing Dark Energy with Baryonic Acoustic Oscillations from Future Large Galaxy Redshift Surveys," Astrophys. J. 598 (2003) 720 arXiv:astro-ph/0307460.

[15] J. R. Pritchard, S. R. Furlanetto and M. Kamionkowski, "Galaxy surveys, inhomogeneous reionization, and dark energy," Mon. Not. Roy. Astron. Soc. 374 (2007) 159 arXiv:astro-ph/0604358.

[16] Z. Haiman, J. J. Mohr and G. P. Holder, "Constraints on quintessence from future galaxy cluster surveys," Astrophys. J. 553 (2000) 545 arXiv:astro-ph/0002336].

[17] A. Refregier, "Weak Gravitational Lensing by Large-Scale Structure," Ann. Rev. Astron. Astrophys. 41 (2003) 645 arXiv:astro-ph/0307212.

[18] R. Jimenez and A. Loeb, "Constraining Cosmological Parameters Based on Relative Galaxy Ages," Astrophys. J. 573 (2002) 37 arXiv:astro-ph/0106145.

[19] B. Chaboyer, "Absolute Ages Of Globular Clusters And The Age Of The Universe," Astrophys. J. 444 (1995) L9 arXiv:astro-ph/9412015.

[20] R. Jimenez, U. G. Jorgensen, P. Thejll and J. MacDonald, "Stellar evolution with mass loss Comparison of numerical and semianalytical computations," Mon. Not. Roy. Astron. Soc. 275 (1995) 1245 arXiv:astro-ph/9502050.

[21] L. M. Krauss and B. Chaboyer, "Age Estimates of Globular Clusters in the Milky Way: Constraints on Cosmology," Science 299 (2003) 65.

[22] R. Jimenez, L. Verde, T. Treu and D. Stern, "Constraints on the equation of state of dark energy and the Hubble constant from stellar ages and the CMB," Astrophys. J. 593, 622 (2003) arXiv:astro-ph/0302560.

[23] J. Simon, L. Verde and R. Jimenez, "Constraints on the redshift dependence of the dark energy potential," Phys. Rev. D 71 (2005) 123001 arXiv:astro-ph/0412269.

[24] M. Sahlen, A. R. Liddle and D. Parkinson, "Direct reconstruction of the quintessence potential," Phys. Rev. D 72 (2005) 083511 arXiv:astro-ph/0506696; L. Samushia and B. Ratra, "Cosmological Constraints from Hubble Parameter versus Redshift Data," Astrophys. J. 650 (2006) L5 arXiv:astro-ph/0607301] ; R. Lazkoz, "Geometrical constraints on dark energy models," AIP Conf. Proc. 960 (2007) 3 [arXiv:0710.2872 [astro-ph]]; N. E. Mavromatos and V. A. Mitsou, "Observational Evidence for Negative-Energy Dust in Late-Times Cosmology," Astropart. Phys. 29 (2008) 442 arXiv:0707.4671 [astro-ph]]; F. C. Carvalho, E. M. Santos, J. S. Alcaniz and J. Santos, "Cosmological Constraints from Hubble Parameter on f(R) Cosmologies," JCAP 0809 (2008) 008 arXiv:0804.2878 [astro-ph]]; E. Fernandez-Martinez and L. Verde, "Prospects in Constraining the Dark Energy Potential," JCAP 0808 (2008) 023 arXiv:0806.1871 [astro-ph]]; A. Kurek, O. Hrycyna and M. Szydlowski, "Constraints on oscillating dark energy models," Phys. Lett. B 659 (2008) 14 arXiv:0707.0292 [astro-ph]]; A. A. Sen and R. J. Scherrer, "The Weak Energy Condition and the Expansion History of the Universe," Phys. Lett. B 659 (2008) 457 arXiv:astro-ph/0703416]; H. Zhang and Z. H. Zhu, "Natural Phantom Dark Energy, Wiggling Hubble Parameter $H(z)$ and Direct $H(z)$ Data," JCAP 0803 (2008) 007 arXiv:astro-ph/0703245; B. Ratra and M. S. Vogeley, "Resource Letter BE-1: The Beginning and Evolution of the Universe," Publ. Astron. Soc. Pac. 120 (2008) 235 arXiv:0706.1565 [astro-ph]];

[25] J. Dunlop, J. Peacock, H. Spinrad, A. Dey, R. Jimenez, D. Stern and R. Windhorst, "A 3.5 Gyr - old galaxy at redshift 1.55," Nature 381 (1996) 581.

[26] H. Spinrad, A. Dey, D. Stern, J. Dunlop, J. Peacock, R. Jimenez and R. Windhorst, "LBDS 
Cosmic expansion history

53W091: An Old red Galaxy at z=1.552," Astrophys. J. 484 (1997) 581.

arXiv:astro-ph/9702233

[27] L. L. Cowie, A. Songaila and A. J. Barger, "Evidence for a Gradual Decline in the Universal Rest-Frame UV Luminosity Density for z < 1," Astron. J. 118 (1999) 603 arXiv:astro-ph/9904345.

[28] A. Heavens, B. Panter, R. Jimenez and J. Dunlop, "The Complete Star Formation History of the Universe," Nature 428 (2004) 625 arXiv:astro-ph/0403293.

[29] D. Thomas, C. Maraston, R. Bender and C. M. de Oliveira, "The epochs of early-type galaxy formation as a function of environment," Astrophys. J. 621 (2005) 673 arXiv:astro-ph/0410209.

[30] B. Panter, R. Jimenez, A. F. Heavens and S. Charlot, "The star formation histories of galaxies in the Sloan Digital Sky Survey," Mon. Not. Roy. Astron. Soc. 378 (2007) 1550 arXiv:astro-ph/0608531.

[31] T. Treu et al., "The Assembly History of Field Spheroidals: Evolution of Mass-to-light Ratios and Signatures of Recent Star Formation," Astrophys. J. 633, 174 (2005) arXiv:astro-ph/0503164.

[32] G. Bruzual and S. Charlot, "Stellar population synthesis at the resolution of 2003," Mon. Not. Roy. Astron. Soc. 344 (2003) 1000 arXiv:astro-ph/0309134.

[33] S. Charlot, G. Worthey and A. Bressan A., "Uncertainties in the Modeling of Old Stellar Populations," Astrophys. J. 457, 625 (1996).

[34] C. Maraston, G. Stromback, D. Thomas, D. A. Wake and R. C. Nichol, "Modeling the color evolution of luminous red galaxies - improvements with empirical stellar spectra," arXiv:0809.1867 [astro-ph].

[35] C. Maraston, "Evolutionary population synthesis: models, analysis of the ingredients and application to high-z galaxies," Mon. Not. Roy. Astron. Soc. 362 (2005) 799 arXiv:astro-ph/0410207.

[36] R. Jimenez, P. Padoan, F. Matteucci and A. F. Heavens, "Galaxy Formation and Evolution: Low Surface Brightness Galaxies," Mon. Not. Roy. Astron. Soc. 299 (1998) 123 arXiv:astro-ph/9804049.

[37] R. Jimenez, J. MacDonald, J. S. Dunlop, P. Padoan and J. A. Peacock, "Synthetic stellar populations: single stellar populations, stellar interior models and primordial proto-galaxies," Mon. Not. Roy. Astron. Soc. 349 (2004) 240 arXiv:astro-ph/0402271].

[38] G. O. Abell, "The Distribution Of Rich Clusters Of Galaxies," Astrophys. J. Suppl. 3 (1958) 211.

[39] P. Rosati, R. Della Ceca, C. Norman and R. Giacconi, "The ROSAT Deep Cluster Survey: The X-Ray Luminosity Function Out To $z=0.8$," Astrophys. J. 492 (1998) L21 arXiv:astro-ph/9710308.

[40] H. Ebeling, A. C. Edge and J. P. Henry, "MACS: A Quest for the Most Massive Galaxy Clusters in the Universe," Astrophys. J. 553 (2001) 668.

[41] P. R. M. Eisenhardt et al., Astrophys. J. 684 (2008) 905 arXiv:0804.4798 [astro-ph]].

[42] S. A. Stanford, B. P. Holden, P. Rosati, P. R. Eisenhardt, D. Stern, G. Squires and H. Spinrad, "An X-ray Selected Galaxy Cluster at $\mathrm{z}=1.11$ in the Rosat Deep Cluster Survey," Astron. J. 123 (2002) 619 arXiv:astro-ph/0110709.

[43] P. Massey and C. Gronwall, "The Kit Peak specotrophotometric standards-Extension to 1 micron," Astrophys. J. 358 (1990) 344.

[44] D. Stern et al., "First Results from the SPICES Survey," arXiv:astro-ph/0012146

[45] Le Fèvre O. et al., "The VIMOS VLT deep survey. First epoch VVDS-deep survey: 11564 spectra with $17.5 \leq \mathrm{IAB} \leq 24$, and the redshift distribution over $0 \leq z \leq 5$," Astron. Astrophys. 439 (2005) 845.

[46] R. Jimenez, L. Verde, T. Treu and D. Stern, "Constraints on the equation of state of dark energy and the Hubble constant from stellar ages and the CMB," Astrophys. J. 593 (2003) 622. arXiv:astro-ph/0302560.

[47] A. G. Riess et al., "A Redetermination of the Hubble Constant with the Hubble Space Telescope from a Differential Distance Ladder," Astrophys. J. 699 (2009) 539 arXiv:0905.0695 [astro-ph.CO]].

[48] Komatsu E. et al., arXiv:0803.0547 E. Komatsu et al. [WMAP Collaboration], "Five-Year Wilkinson Microwave Anisotropy Probe (WMAP) Observations:Cosmological Interpretation," Astrophys. J. Suppl. 180, 330 (2009) arXiv:0803.0547[astro-ph]].

[49] J. Dunkley et al. [WMAP Collaboration], "Five-Year Wilkinson Microwave Anisotropy Probe (WMAP) Observations: Likelihoods and Parameters from the WMAP data," Astrophys. J. Suppl. 180 (2009) 306 arXiv:0803.0586 [astro-ph]]. 
[50] D. G. Figueroa, L. Verde and R. Jimenez, "Improved Cosmological Parameter Constraints from CMB and H(z) Data," JCAP 0810 (2008) 038 arXiv:0807.0039 [astro-ph]].

[51] P. Coelho, G. Bruzual, S. Charlot, A. Weiss, B. Barbuy and J. Ferguson, "Spectral models for solar scaled and alpha-enhanced stellar populations," Mon. Not. Roy. Astron. Soc. 382 (2007) 498 arXiv:0708.2790 [astro-ph]].

[52] Wolf, M. J., Drory, N., Gebhardt, K., Hill, G. J. "Ages and Metallicities of Extragalactic Globular Clusters from Spectral and Photometric Fits of Stellar Population Synthesis Models". Astrophysical Journal 655 (2007) 179-211

[53] C. J. Walcher, P. Coelho, A. Gallazzi and S. Charlot, arXiv:0906.5000 [astro-ph.CO]. 